\title{
Biodiversidade de Ichneumonidae (Hymenoptera) \\ e monitoramento das espécies de Pimplinae e Poemeniinae do Capão da Imbuia, Curitiba, Paraná ${ }^{1}$
}

\author{
Alice Fumi Kumagai ${ }^{2}$ \\ Vinalto Graf $^{3}$
}

\begin{abstract}
Biodiversity of Ichneumonidae (Hymenoptera) and monitoring of the species of Pimplinae and Poemeniinae from Capão da Imbuia, Curitiba, Paraná. The ichneumofauna of Capão da Imbuia was studied during three years of capture, using Malaise traps. 114,469 specimens were captured, of which 3,603 belong to 20 subfamilies of ichneumonids. Pimplinae and Poemeniinae in the wood contains 14 genera and 48 species. In the first sample (1978-1979), 23 species were captured; in the second (1985-1986), 21 species were obtained, 13 of which had been previously captured, with an addition of eight species. And in the last sample (1995-1996), 39 species were obtained, of which 17 had not previously been captured. The dominant species, considering the number of individuals, were: Pimpla semirufa Brullé, 1846, Pimpla caerulea Brullé, 1846, Neotheronia aff. hespenheidei, Pimpla golbachi (Porter, 1970), Pimpla sumichrasti Cresson, 1874, Flacopimpla sulina Graf \& Kumagai, 1997 and Pimpla tomyris Schrottky, 1902.

KEY WORDS. Hymenoptera, Ichneumonidae, Pimplinae, Poemeniinae, monitoring, species richness
\end{abstract}

Dentre os Ichneumonidae, as subfamílias Pimplinae, Poemeniinae, Rhyssinae, Acaenitinae e Cylloceriinae formam o grupo dos pimpliformes inferiores (sensu GAULD 1991). Pimplinae possui aproximadamente 60 gêneros descritos e são mais ricos em espécies em áreas tropicais (GASTON \& GAULD 1993). A biologia dos pimplines é bastante variada e podem ser ectoparasitóides idiobiontes de hospedeiros não expostos, ectoparasitóides coinobiontes de aranhas, endoparasitóides idiobiontes de prepupas e pupas de Lepidoptera. Alguns pimplines são parasitóides comuns de insetos-pragas de culturas economicamente importantes, mas em geral não possuem especificidade de hospedeiro, o que resulta em pouca utilização destas espécies em controle biológico. E Poemeniinae é uma pequena subfamília com dez gêneros (GAULD 1991). Suas espécies são ectoparasitóides idiobiontes de Cerambycidae, Curculionidae, Melandryidae e também Sphecidae. Em TownES \& TOWNES (1966) apenas Ganodes Townes, 1957 é citado para a região Neotropical. Em 1991 uma nova espécie é descrita para a Costa Rica, Ganodes matai Gauld, 1991 e também um novo gênero, Rodrigama (R. gamezi Gauld, 1991).

1) Contribuição número 1311 do Departamento de Zoologia, Universidade Federal do Paraná.

2) Departamento de Zoologia, Universidade Federal de Minas Gerais. Caixa Postal 486, 31270-901 Belo Horizonte, Minas Gerais, Brasil. E-mail: acfk@ icb.ufmg.br

3) Departamento de Zoologia, Universidade Federal do Paraná. Caixa Postal 19020, 81531-980 Curitiba, Paraná, Brasil. E-mail: vig@bio.ufpr.br 
Neste trabalho a biodiversidade de Ichneumonidae do Capão da Imbuia é estudada em três fases de amostragem, com intervalos de seis e quinze anos após a primeira (1978-1979), assim como a abundância e riqueza das espécies de Pimplinae e Poemeniinae

\section{MATERIAL E MÉTODOS}

\section{Local de amostragem}

As plantas do Capão da Imbuia foram listadas por DoMBRowsKI \& KUNIYOSHI (1967) e a descrição da área de amostragem foi feita por KUMAGAI \& GRAF (2000) quando comparou a fauna de Ichneumonidae do bosque com uma área rural na região metropolitana de Curitiba.

\section{Amostragem}

A fauna de Ichneumonidae do Capão da Imbuia foi amostrada no período de 19.V.1978 a 30.VI.1979 (= CR78) (KUMAGAI \& GRAF 2000). Novas coletas foram feitas após seis (4.VII.1985 a 3.VII.1986=CR85) e 15 anos (17.V.1995 a 17.V.1996 = CR95). A metodologia empregada foi a mesma, ou seja, o uso de armadilha Malaise (modelo TOWNES 1972), igual periodicidade na retirada das amostras (semanal) e no mesmo local. Nas duas primeiras amostragens (CR78 e CR85) o bosque era de acesso apenas para pesquisadores e funcionários. A visitação pública teve início à partir de novembro de 1992, quando foram feitas algumas alterações: uma passarela metálica a $0,5 \mathrm{~m}$ do solo sobre a trilha que corta o bosque; uma fonte com queda-d'água; dioramas com animais e plantas que existiam no local e alguns mamíferos foram introduzidos. E também, desde a primeira amostragem houve um aumento significativo de urbanização nas áreas próximas ao bosque. As temperaturas médias anuais (máxima) foram semelhantes em CR85 $\left(24,19^{\circ} \mathrm{C}\right)$ e CR95 $\left(23,63^{\circ} \mathrm{C}\right)$ e um pouco menor em CR78 $\left(22,10^{\circ} \mathrm{C}\right)$, as temperaturas médias anuais mínimas foram semelhantes em CR78 $\left(13,42^{\circ} \mathrm{C}\right)$ e CR95 $\left(13,45^{\circ} \mathrm{C}\right)$ e um pouco menor em CR85 $\left(11,71^{\circ} \mathrm{C}\right)$. Segundo MAACK $(1981)$, o total anual médio da precipitação é $1.451,8 \mathrm{~mm}$ e nos períodos de coleta foram de 1.312,8 $\mathrm{mm}$ (CR78), $1.042,0 \mathrm{~mm}$ (CR85) e 1.614,9 mm (CR95) de chuva.

\section{Análise dos dados}

A análise da distribuição de abundância das espécies foi feito pelo método de Kato, Matsuda \& Yamashita 1952 (in LAROCA 1995). Para o índice de ocorrência e dominância das espécies utilizou-se o método proposto por Palma, 1975 (in ABREU \& NOGUEIRA 1989).

\section{Depósito do material}

Os ichneumonídeos deste trabalho foram depositados no Departamento de Zoologia do Instituto de Ciências Biológicas da Universidade Federal de Minas Gerais (Belo Horizonte, Minas Gerais) (amostras CR85 e CR95) e na Coleção de Entomologia J.S. Moure, do Departamento de Zoologia do Setor de Ciências Biológicas da Universidade Federal do Paraná (Curitiba, Paraná) (amostra CR78). 


\section{RESULTADOS E DISCUSSÃO}

\section{Total de insetos}

Foram capturados um total de 114.407 insetos. Na comparação das amostragens observa-se que CR95 teve uma captura 142,96\% maior do que em CR85 e em relação a CR78 foi $18,26 \%$. Das 16 ordens capturadas (Tab. I), Dermaptera ocorreu somente em CR85; Ephemeroptera; Odonata e Thysanoptera estiveram presentes somente em CR95 e Strepsiptera esteve ausente em CR78. Em KUMAGAI \& GRAF (2000) as ordens mais freqüentes foram Diptera (81,00\%), Hymenoptera $(7,19 \%)$ e Lepidoptera $(3,66 \%)$ com percentuais semelhantes aos encontrados em CR85 (84,42\%; 7,98\% e 3,77\% respectivamente), mas em CR95 os Diptera $(66,78 \%)$ foram menos frequientes e os Collembola $(10,81 \%)$ foram muito mais abundantes que nas duas amostragens precedentes. Os Collembola são insetos de lugares úmidos e a alta frequêencia em CR95 pode ser associada à modificação ocorrida no bosque em 1992 e pela precipitação que foi mais elevada em cerca de $300 \mathrm{~mm}$. Este ambiente úmido também propiciou uma grande proliferação de "beijinhos" (Impatiens sultani Hooker) (Balsaminaceae). Os meses de maior captura de insetos foram em novembro (CR85) e em janeiro (CR95), e nas três amostragens as menores frequiências de indivíduos foram em junho, coincidindo com as mais baixas médias semanais de temperatura $\left(3,58^{\circ}\right.$ a $\left.7,61^{\circ} \mathrm{C}\right)$.

Tabela I. Insetos capturados com armadilha Malaise no Capão da Imbuia, Curitiba, Paraná em (CR78) 1978-1979(KUMAGAI \& GRAF 2000), (CR85) 1985-1986 e (CR95) 1995-1996.

\begin{tabular}{|c|c|c|c|c|}
\hline Ordem & CR78 & CR85 & CR95 & Total \\
\hline Collembola & 426 & 134 & 5.482 & 6.042 \\
\hline Ephemeroptera & - & - & 2 & 2 \\
\hline Odonata & - & - & 2 & 2 \\
\hline Orthoptera & 117 & 43 & 174 & 334 \\
\hline Isoptera & 23 & 11 & 112 & 146 \\
\hline Dermaptera & - & 2 & - & 2 \\
\hline Psocoptera & 580 & 46 & 1.105 & 1.731 \\
\hline Thysanoptera & - & - & 14 & 14 \\
\hline Hemiptera & 181 & 75 & 109 & 365 \\
\hline Homoptera & 1.234 & 165 & 826 & 2.225 \\
\hline Neuroptera & 19 & 5 & 76 & 100 \\
\hline Coleoptera & 907 & 313 & 1.497 & 2.717 \\
\hline Strepsiptera & - & 2 & 1 & 3 \\
\hline Lepidoptera & 1.570 & 787 & 2.460 & 4.817 \\
\hline Diptera & 34.717 & 17.613 & 34.867 & 87.197 \\
\hline Hymenoptera & 3.084 & 1.666 & 3.960 & 8.710 \\
\hline Total & 42.858 & 20.862 & 50.687 & 114.407 \\
\hline
\end{tabular}

\section{Total de Ichneumonidae}

As maiores capturas de Ichneumonidae foram nas amostragens de CR78, CR95 e CR85, com respectivamente 1.363, 1.281 e 959 indivíduos. Das 20 subfamílias que ocorreram no bosque, 16 estão presentes nas três amostragens, sendo que Ctenopelmatinae e Metopiinae não foram capturadas em CR78 e CR85 respectivamente. Xoridinae esteve presente somente em CR85 e Lycorininae em CR95. Cryptinae foi a subfamília mais freqüente em CR78 e CR85 com respectivamente 411 e 391 indivíduos, mas em CR95 foi diferente pois Pimplinae foi a mais freqüente (376 indivíduos) seguida de Cryptinae (263 indivíduos) (Tab. II). A maior 
captura de ichneumonídeos em CR78 e CR85 foi em novembro e em CR95 ocorreu em dezembro e a menor foi em junho para as três amostragens, mas em CR85 a captura foi muito menor que nas outras duas. A flutuação mensal da captura dos ichneumonídeos foi semelhante em CR78 e CR85, mas esta última apresentou uma menor freqüência de indivíduos durante todo o ano, exceto em agosto quando CR78 possui a metade da captura de CR85. E CR95 apresentou uma maior freqüência, em relação as duas outras amostragens, nos meses de setembro e dezembro e menor em novembro, justo quando ocorreu o pico de freqüência em CR78 e CR85. De abril em diante CR85 e CR95 tiveram menos indivíduos que CR78 (Tab. III).

Tabela II. Ichneumonídeos capturados com armadilha Malaise no Capão da Imbuia, Curitiba, Paraná em (CR78) 1978-1979(KUMAGAI \& GRAF 2000), (CR85) 1985-1986 e (CR95) 1995-1996.

\begin{tabular}{|c|c|c|c|c|}
\hline Subfamília & CR78 & CR85 & CR95 & Total \\
\hline Pimplinae & 133 & 111 & 376 & 620 \\
\hline Poemeniinae & 1 & 2 & 1 & 4 \\
\hline Tryphoninae & 10 & 44 & 65 & 119 \\
\hline Labeninae & 1 & 3 & 1 & 5 \\
\hline Xoridinae & - & - & 1 & 1 \\
\hline Cryptinae & 411 & 391 & 263 & 1.065 \\
\hline Lycorininae & - & - & 1 & 1 \\
\hline Banchinae & 12 & 15 & 36 & 63 \\
\hline Ctenopelmatinae & - & 1 & 4 & 5 \\
\hline Campopleginae & 98 & 120 & 73 & 291 \\
\hline Cremastinae & 10 & 1 & 17 & 28 \\
\hline Tersilochinae & 40 & 4 & 15 & 59 \\
\hline Ophioninae & 4 & 29 & 67 & 100 \\
\hline Mesochorinae & 74 & 14 & 20 & 108 \\
\hline Metopinae & 5 & - & 6 & 11 \\
\hline Anomaloninae & 1 & 2 & 6 & 9 \\
\hline Microleptinae & 34 & 8 & 7 & 49 \\
\hline Orthocentrinae & 268 & 28 & 101 & 397 \\
\hline Diplazontinae & 26 & 1 & 1 & 28 \\
\hline Ichneumoninae & 235 & 184 & 221 & 640 \\
\hline Total & 1.363 & 959 & 1.281 & 3.603 \\
\hline
\end{tabular}

Tabela III. Captura mensal de ichneumonídeos com armadilha Malaise no Capão da Imbuia, Curitiba, Paraná em (CR78) 1978-1979(KUMAGAI \& GRAF 2000), (CR85) 1985-1986 e (CR95) 1995-1996.

\begin{tabular}{|c|c|c|c|c|}
\hline Mês & CR78 & CR85 & CR95 & Total \\
\hline Maio & 7 & - & 6 & 13 \\
\hline Junho & 40 & - & 42 & 82 \\
\hline Julho & 48 & 7 & 71 & 126 \\
\hline Agosto & 68 & 135 & 131 & 334 \\
\hline Setembro & 58 & 64 & 177 & 299 \\
\hline Outubro & 202 & 119 & 136 & 457 \\
\hline Novembro & 243 & 199 & 126 & 568 \\
\hline Dezembro & 196 & 178 & 241 & 615 \\
\hline Janeiro & 168 & 161 & 133 & 462 \\
\hline Fevereiro & 110 & 45 & 73 & 228 \\
\hline Março & 63 & 18 & 72 & 153 \\
\hline Abril & 54 & 16 & 53 & 123 \\
\hline Maio & 64 & 10 & 20 & 94 \\
\hline Junho & 42 & 6 & - & 48 \\
\hline Julho & - & 1 & - & 1 \\
\hline Total & 1.363 & 959 & 1.281 & 3.603 \\
\hline
\end{tabular}




\section{Total de gêneros e espécies de Pimplinae e Poemeniinae}

Em CR78 (KumAGAI \& GRAF 2000) foram capturados 134 indivíduos, enquanto que em CR85 foram 113 e em CR95, 377 indivíduos. A freqüência foi maior em CR95, com 29,43\% dos ichneumonídeos capturados, em CR85 com $11,78 \%$ e CR78 com 9,83\%. Nas três amostragens foram capturados um total de 13 gêneros de Pimplinae (47 espécies) e um de Poemeniinae (somente a espécie Ganodes balteatus Townes, 1957). Seis gêneros são comuns às três amostragens (Zonopimpla Ashmead, 1900, Polysphincta Gravenhorst, 1829, Neotheronia Krieger, 1899, Itoplectis Foerster, 1868, Pimpla Fabricius, 1804 e Ganodes); dois ocorreram em CR85 e CR95 (Tromatobia Foerster, 1868 e Apechthis Foerster, 1868) e três em CR78 e CR95 (Clistopyga Gravenhorst, 1829, Eruga Townes, 1960 e Zatypota Foerster, 1869). Os gêneros Dolichomitus Smith, 1877 e Hymenoepimecis Viereck, 1912 foram capturados somente em CR85 e Flacopimpla Gauld, 1991 esteve presente apenas em CR95. Os gêneros mais ricos em espécies foram Pimpla e Neotheronia, sendo que em CR95 o mais rico foi Neotheronia com dez espécies e depois foi Pimpla, com sete (Tab. IV). Das 48 espécies presentes no bosque, duas ocorreram somente na amostragem de CR78 (Polysphincta thoracica (Cresson, 1874) e Pimpla croceiventris (Cresson, 1868)); em CR85 foram seis espécies (Dolichomitus sp. 3, Tromatobia sp. 2, Ephialtini sp. 1, Hymenoepimecis aff. cameroni, Neotheronia sp. 1 e Pimpla sp.1) e em CR95 foram 17 (Fig. 1).

As espécies consideradas dominantes pelo método de Kato são quatro em CR78 (Pimpla semirufa Brullé, 1846, Pimpla caerulea Brullé, 1846, Neotheronia aff. hespenheidei e Pimpla golbachi (Porter, 1970)), quatro em CR85 (Pimpla tomyris Schrottky, 1902, Pimpla sumichrasti Cresson, 1874, Neotheronia aff. hespenheidei e Pimpla semirufa) e seis em CR95 (Pimpla semirufa, Pimpla golbachi, Neotheronia aff. hespenheidei, Pimpla caerulea, Pimpla sumichrasti e Flacopimpla sulina Graf \& Kumagai, 1997), esta última espécie foi coletada somente em CR95 (17 exemplares), era nova para a fauna brasileira e sua distribuição foi ampliada para o sudeste e sul do Brasil (KUMAGAI \& GRAF 1997). E duas espécies (Neotheronia aff. hespenheidei e Pimpla semirufa) foram consideradas dominantes nas três amostragens (Tab. V).

Na classificação de Palma, para a ocorrência e dominância das espécies, as consideradas raras ocorreram em maior número na amostragem de CR95 (32 espécies), depois foi em CR78 e CR85 com respectivamente 14 e 12 espécies, em CR78 e CR85 foram 9 espécies intermediárias e CR95 teve 7 e não houve espécie comum no Capão da Imbuia (Tabs IV e V). Apesar de mais freqüentes, as espécies Pimpla semirufa (105 indivíduos) e Pimpla golbachi (56 indivíduos) não foram consideradas comuns devido às capturas estarem concentradas nos meses de agosto à dezembro.

Em 1978 (Kumagai \& GRAF 2000) foram capturadas 22 espécies de Pimplinae e uma de Poemeniinae. Em CR85 foram 21 espécies, com oito espécies não capturadas anteriormente. Na amostragem de CR95 obteve-se 39 espécies, das quais 17 ainda não haviam sido capturadas nas duas amostragens anteriores (Fig. 
Tabela IV. Abundância das espécies de Pimplinae e Poemeniinae capturadas no Capão da Imbuia, Curitiba, Paraná em (CR78) 1978-1979(KUMAGAI \& GRAF 2000), (CR85) 1985-1986 e (CR95) 1995-1996.

\begin{tabular}{|c|c|c|c|c|}
\hline Espécies & CR78 & CR85 & CR95 & Total \\
\hline \multicolumn{5}{|l|}{ Pimplinae } \\
\hline \multicolumn{5}{|l|}{ Ephialtini } \\
\hline Dolichomitus sp. 3 & & 2 & & 2 \\
\hline Zonopimpla lilae Gauld, 1991 & & & 1 & 1 \\
\hline Zonopimpla sp. 1 & & & 2 & 2 \\
\hline Zonopimpla sp. 4 & 1 & & 3 & 4 \\
\hline Zonopimpla sp. 5 & & & 3 & 3 \\
\hline Zonopimpla sp. 6 & & & 1 & 1 \\
\hline Zonopimpla sp. 8 & 1 & 1 & & 2 \\
\hline Tromatobia sp. 2 & & 2 & & 2 \\
\hline Tromatobia sp. 7 & & & 1 & 1 \\
\hline Clistopyga jakobi Graf, 1985 & $5^{*}$ & & 1 & 6 \\
\hline Ephialtini sp. 1 & & 1 & & 1 \\
\hline \multicolumn{5}{|l|}{ Polysphinctini } \\
\hline Polysphincta thoracica (Cresson, 1874) & $8^{*}$ & & & 8 \\
\hline Polysphincta sp. 1 & & & 2 & 2 \\
\hline Polysphincta sp. 4 & & 1 & 1 & 2 \\
\hline Polysphincta sp. 5 & & & 3 & 3 \\
\hline Polysphincta sp. 7 & & & 1 & 1 \\
\hline Hymenoepimecis aff. cameroni & & 1 & & 1 \\
\hline Eruga draperi Gauld, 1991 & 1 & & 1 & 2 \\
\hline Flacopimpla sulina Graf \& Kumagai, 1997 & & & $17^{*}$ & 17 \\
\hline Zatypota alborhombarta (Davis, 1895) & $6^{*}$ & & 9 & 15 \\
\hline Zatypota sp. 1 & 2 & & 2 & 4 \\
\hline Zatypota sp. 2 & & & 3 & 3 \\
\hline Zatypota sp. 3 & & & 1 & 1 \\
\hline \multicolumn{5}{|l|}{ Pimplini } \\
\hline Neotheronia aff. hespenheidei & $15^{*}$ & $12^{*}$ & $40^{*}$ & 67 \\
\hline Neotheronia lineata (Fabricius, 1804) & 3 & $6^{*}$ & $12^{*}$ & 21 \\
\hline Neotheronia tacubaya (Cresson, 1874) & 1 & 2 & 4 & 7 \\
\hline Neotheronia lloydi Gauld, 1991 & & & 10 & 10 \\
\hline Neotheronia concolor Krieger, 1905 & 1 & & 6 & 7 \\
\hline Neotheronia aff. donovani & & & 1 & 1 \\
\hline Neotheronia chiriquensis (Cameron, 1886) & 2 & & 3 & 5 \\
\hline Neotheronia tolteca (Cresson, 1874) & & & 5 & 5 \\
\hline Neotheronia sp. 1 & & 1 & & 1 \\
\hline Neotheronia sp. 4 & & & 1 & 1 \\
\hline Neotheronia sp. 5 & & & 1 & 1 \\
\hline Apechthis sp. 1 & & 1 & 2 & 3 \\
\hline Apechthis sp. 2 & & & 3 & 3 \\
\hline Itoplectis lissa Porter, 1970 & 1 & 4 & 1 & 6 \\
\hline Itoplectis brasiliensis (Dalla Torre, 1901) & 1 & 1 & 2 & 4 \\
\hline Pimpla sumichrasti Cresson, 1874 & $6^{*}$ & $15^{*}$ & $23^{*}$ & 44 \\
\hline Pimpla azteca Cresson, 1874 & 1 & & 2 & 3 \\
\hline Pimpla caerulea Brullé, 1846 & $21^{*}$ & $8^{*}$ & $37^{*}$ & 66 \\
\hline Pimpla tomyris Schrottky, 1902 & $7^{*}$ & $30^{*}$ & 5 & 42 \\
\hline Pimpla croceiventris (Cresson, 1868) & 2 & & & 2 \\
\hline Pimpla trichroa (Porter, 1970) & 2 & $5^{*}$ & 5 & 12 \\
\hline Pimpla golbachi (Porter, 1970) & $12^{*}$ & $6^{*}$ & $56^{*}$ & 74 \\
\hline Pimpla semirufa Brullé, 1846 & $34^{*}$ & $10^{*}$ & 105 & 149 \\
\hline Pimpla sp. 1 & & 2 & & 2 \\
\hline \multicolumn{5}{|l|}{ Poemeniinae } \\
\hline Ganodes balteatus Townes, 1957 & 1 & 2 & 1 & 4 \\
\hline Total & 134 & 113 & 377 & 624 \\
\hline
\end{tabular}

(*) Espécies intermediárias segundo a classificação de Palma e todas as outras são raras.

Revta bras. Zool. 19 (2): 445 - 452, 2002 
1978-79

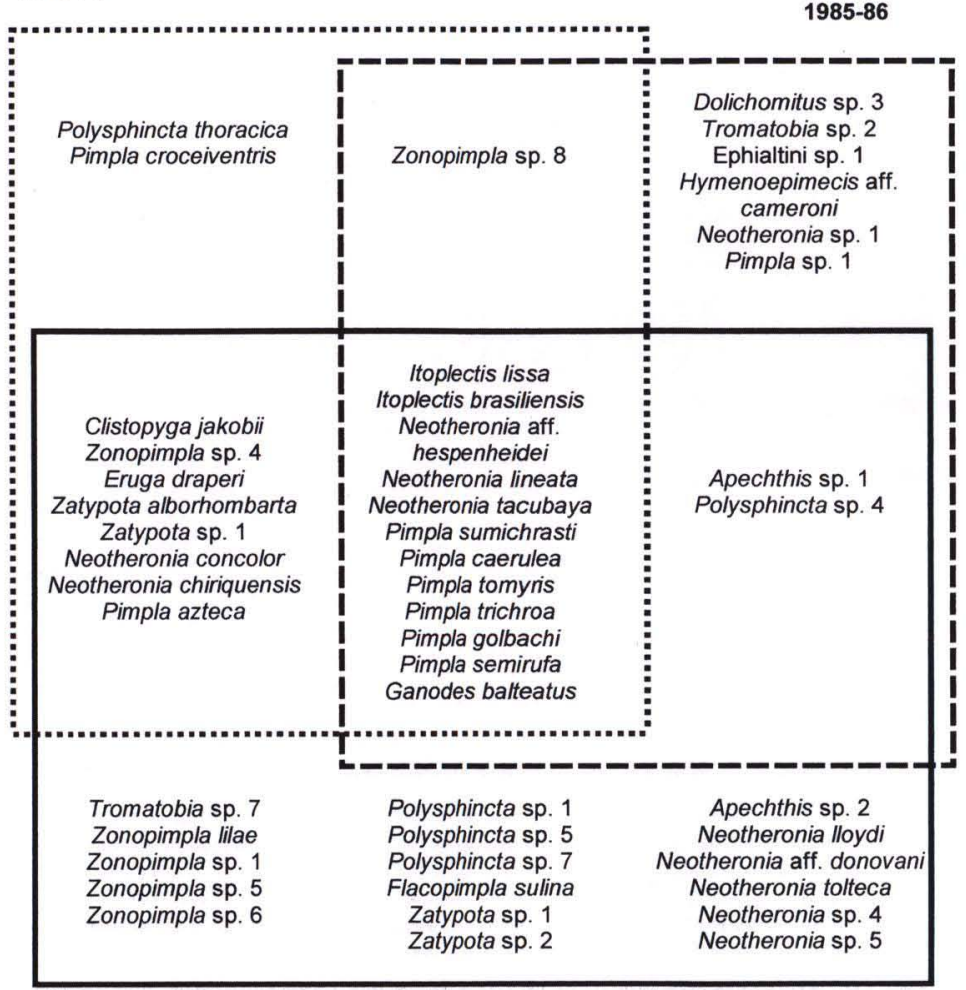

1995-96

Fig. 1. Espécies de Pimplinae e Poemeniinae capturadas com armadilha de Malaise no Capão da Imbuia, Curitiba, Paraná, nos anos de 1978-1979 (Kumagal \& Graf 2000 = CR78), 1985-1986 (CR85) e 1995-1996 (CR95).

1). Em CR95 destacam-se Flacopimpla sulina (17 indivíduos) e Neotheronia lloydi Gauld, 1991 (dez indivíduos) com alta frequiência e só capturadas nesta amostragem, e as espécies Neotheronia aff. hespenheidei, Neotheronia lineata (Fabricius, 1804), Pimpla sumichrasti, Pimpla caerulea, Pimpla golbachi e Pimpla semirufa que foram mais freqüentes do que nas duas amostragens anteriores.

A fauna do Capão da Imbuia é numericamente menor que a da Costa Rica (GAULD 1991), onde 144 espécies foram capturadas em 17 locais em diferentes altitudes e a utilização de mais de 100 armadilhas Malaise. Mas a repetição de amostragem mostrou um significativo aumento no número de espécies no Capão da Imbuia. Podemos concluir então, que a armadilha Malaise foi um eficiente meio de captura de Ichneumonidae, mas mais amostragens são necessárias para que a curva assintótica das espécies possa ser alcançada. 
Tabela V. Distribuição sazonal e freqüência das espécies dominantes (método de Kato) de Pimplinae no Capão da Imbuia, Curitiba, Paraná em (CR78) 1978-1979(KumAGAI \& GRAF 2000), (CR85) 1985-1986 e (CR95) 1995-1996.

\begin{tabular}{|c|c|c|c|c|c|c|c|c|c|c|c|c|c|c|}
\hline \multirow{2}{*}{ Espécies } & \multicolumn{13}{|c|}{ Meses } & \multirow{2}{*}{ Total } \\
\hline & & Jan & Fev & Mar & Abr & Mai & Jun & Jul & Ago & Set & Out & Nov & Dez & \\
\hline \multirow{3}{*}{ Pimpla semirufa } & CR78 & 1 & - & 1 & - & - & 3 & 4 & 3 & - & 8 & 8 & 6 & 34 \\
\hline & CR85 & 2 & - & - & - & - & - & - & 3 & - & - & 4 & 1 & 10 \\
\hline & CR95 & 2 & - & 2 & - & - & - & 2 & 17 & 43 & 22 & 3 & 14 & 105 \\
\hline \multirow{3}{*}{ Neotheronia aff. hespenheidei } & CR78 & - & 1 & 3 & 1 & 4 & 1 & 1 & 2 & - & 1 & - & 1 & 15 \\
\hline & CR85 & 1 & 1 & - & 1 & 1 & - & - & 5 & 1 & 1 & 1 & - & 12 \\
\hline & CR95 & 8 & 4 & 6 & 8 & - & 1 & 1 & 2 & 2 & 1 & 3 & 4 & 40 \\
\hline \multirow[t]{2}{*}{ Pimpla caerulea } & CR78 & - & 1 & 1 & 1 & - & 1 & 4 & 1 & 1 & 3 & 5 & 3 & 21 \\
\hline & CR95 & - & - & 4 & 1 & - & 2 & 5 & 6 & 5 & 4 & 4 & 6 & 37 \\
\hline \multirow[t]{2}{*}{ Pimpla golbachi } & CR78 & - & - & - & - & 1 & - & 1 & 2 & 1 & 3 & 3 & 1 & 12 \\
\hline & CR95 & 2 & - & 1 & - & - & 2 & 1 & 6 & 26 & 6 & 1. & 11 & 56 \\
\hline \multirow[t]{2}{*}{ Pimpla sumichrasti } & CR85 & 4 & - & - & - & - & - & - & 3 & - & 4 & 1 & 3 & 15 \\
\hline & CR95 & 2 & 2 & - & 4 & - & - & 1 & 3 & - & 2 & 4 & 5 & 23 \\
\hline Pimpla tomyris & CR85 & 4 & - & 1 & - & - & - & - & 3 & 3 & 8 & 6 & 5 & 30 \\
\hline Flacopimpla sulina & CR95 & 3 & 2 & - & 1 & 1 & 1 & 3 & 1 & - & - & 2 & 3 & 17 \\
\hline
\end{tabular}

AGRADECIMENTOS. À Prefeitura Municipal de Curitiba, Museu de História Natural Capão da Imbuia, pela permissão de coleta no bosque; à Solange R. Malkowski, entomóloga do Museu de História Natural Capão da Imbuia, pela ajuda nas coletas; à Profa Danúncia Urban, Departamento de Zoologia, UFPR pela leitura crítica dos originais, ao Prof. Olavo Guimarães, Departamento de Botânica, UFPR pela identificação da planta "beijinhos" (Impatiens sultani Hooker) (Balsaminaceae).

\section{REFERÊNCIAS BIBLIOGRÁFICAS}

AbReU, P.C.O.V.\& C.R. NogUeIRA. 1989. Spatial distribution of Siphonophora species at Rio de Janeiro coast, Brazil. Ciênc. Cult. 41 (9): 897-902.

DombrowSKI, L.T.D. \& Y.S. KUNIYOSHI. 1967. A vegetação do "Capão da Imbuia”. I. Araucariana (Botânica), Curitiba, 1: 1-18.

GASTON, K.J. \& I.D. GAULD. 1993. How many species of pimplines (Hymenoptera: Ichneumonidae) are there in Costa Rica? Jour. Trop. Ecol. 9: 491-499.

Gauld, I.D. 1991. The Ichneumonidae of Costa Rica. Mem. Amer. Entomol. Inst., Gainesville, 47: $1-589$.

Kumagai, A.F. \& V. Graf, 1997. A ocorrência de Flacopimpla Gauld no Brasil (Hymenoptera, Ichneumonidae, Pimplinae). Revta bras. Zool. 14 (4): 773-777.

- 2000. Ichneumonidae (Hymenoptera) de áreas urbana e rural, em Curitiba - Paraná, Brasil.

Acta Biol. Paranaense, Curitiba, 28 (1,2,3,4): 153-168.

LAROCA, S. 1995. Ecologia - princípios e métodos. Petrópolis, Ed. Vozes, 197p.

MAACK, R. 1981. Geografia Física do Estado do Paraná. Rio de Janeiro, José Olympio Editora, 450p.

Townes, H. 1972. A light-weight Malaise trap. Entomol. News 83 (9): 239-247.

Townes, H. \& M. Townes. 1966. A catalog and reclassification of the Neotropic Ichneumonidae. Mem.

Amer. Entomol. Inst. 8: 1-367.

Recebido em 21.IX.2001; aceito em 07.V.2002.

Revta bras. Zool. 19 (2): 445 - 452, 2002 